\title{
Malignant Mesenchymoma
}

National Cancer Institute

\section{Source}

National Cancer Institute. Malignant Mesenchymoma. NCI Thesaurus. Code C4268.

A term describing a malignant soft tissue tumor which consists of two or more

mesenchymal lines of differentiation, excluding a fibroblastic line of differentiation. 